\title{
Credit and Debit Card Interchange Fees in Various Countries
}

\section{August 2020 Update}

\author{
Payments System Research ${ }^{\dagger}$
}

Federal Reserve Bank of Kansas City

${ }^{\dagger}$ Fumiko Hayashi, Payments Policy Advisor and Economist, and Rebecca Ruiz, Research Associate, compiled this update. The original charts appeared in Hayashi, Fumiko, 2010. "Payment Card Interchange Fees and Merchant Service Charges - An International Comparison,” Lydian Payments Journal, 1(3): 6-22. 


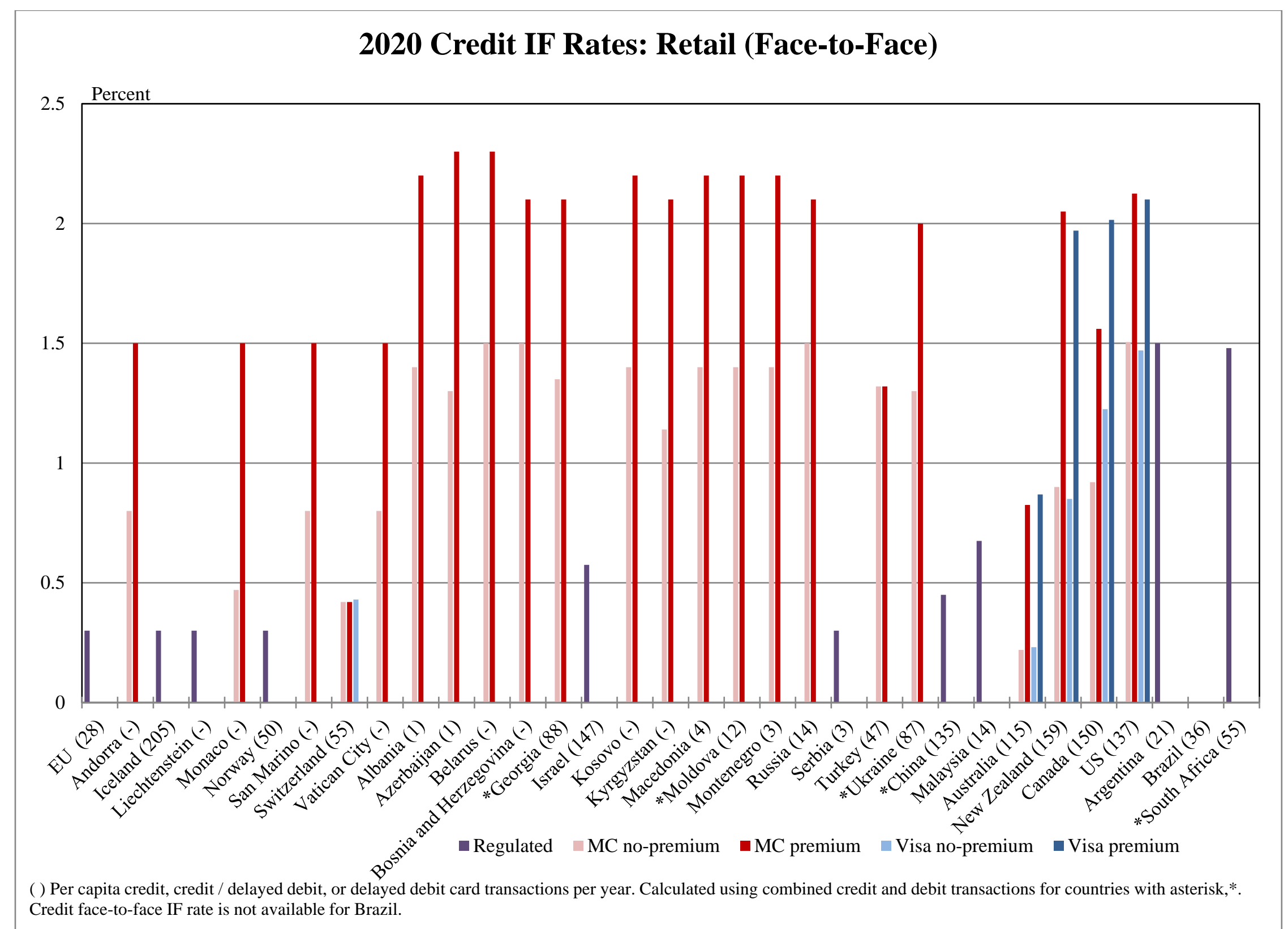




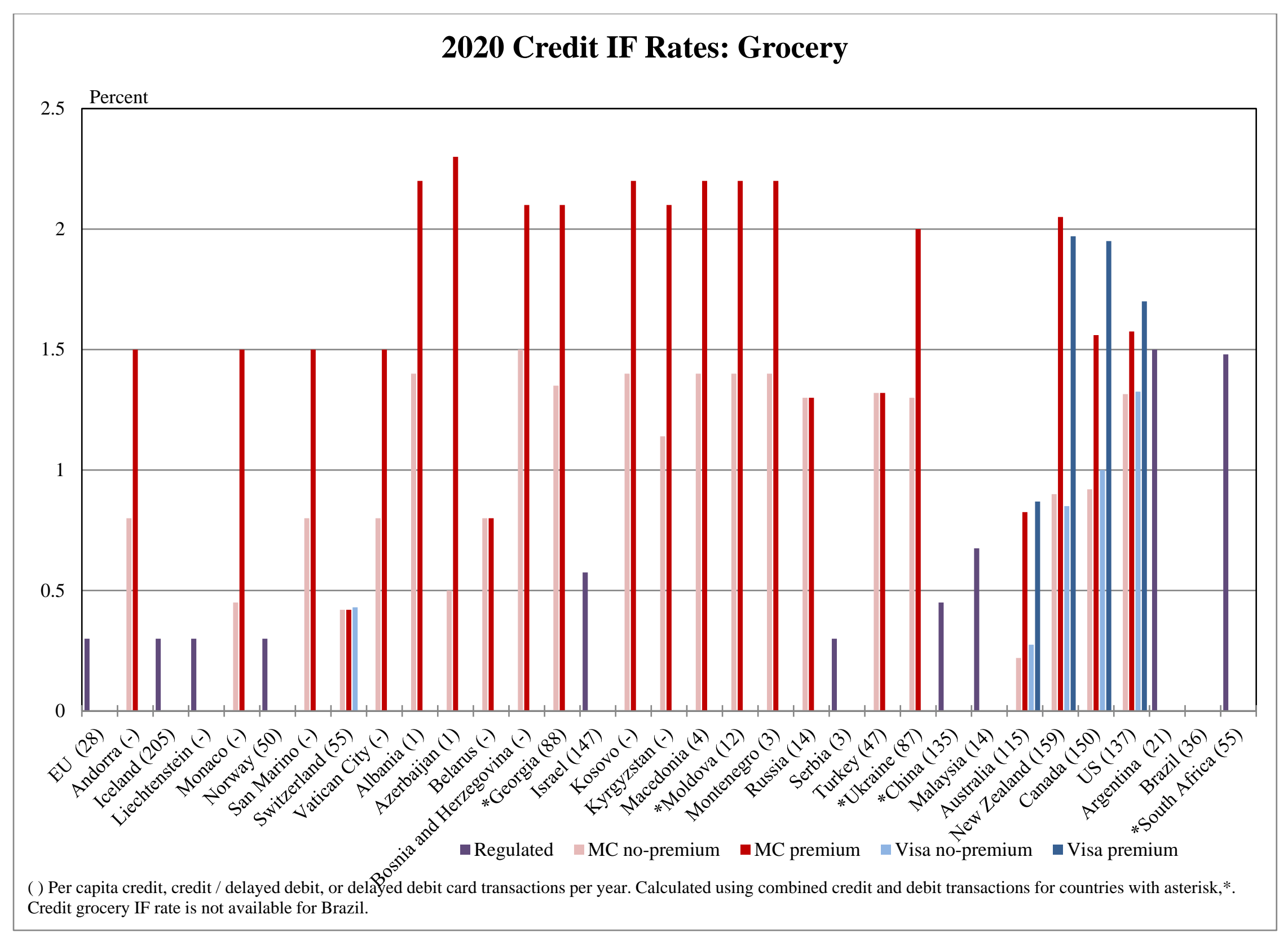




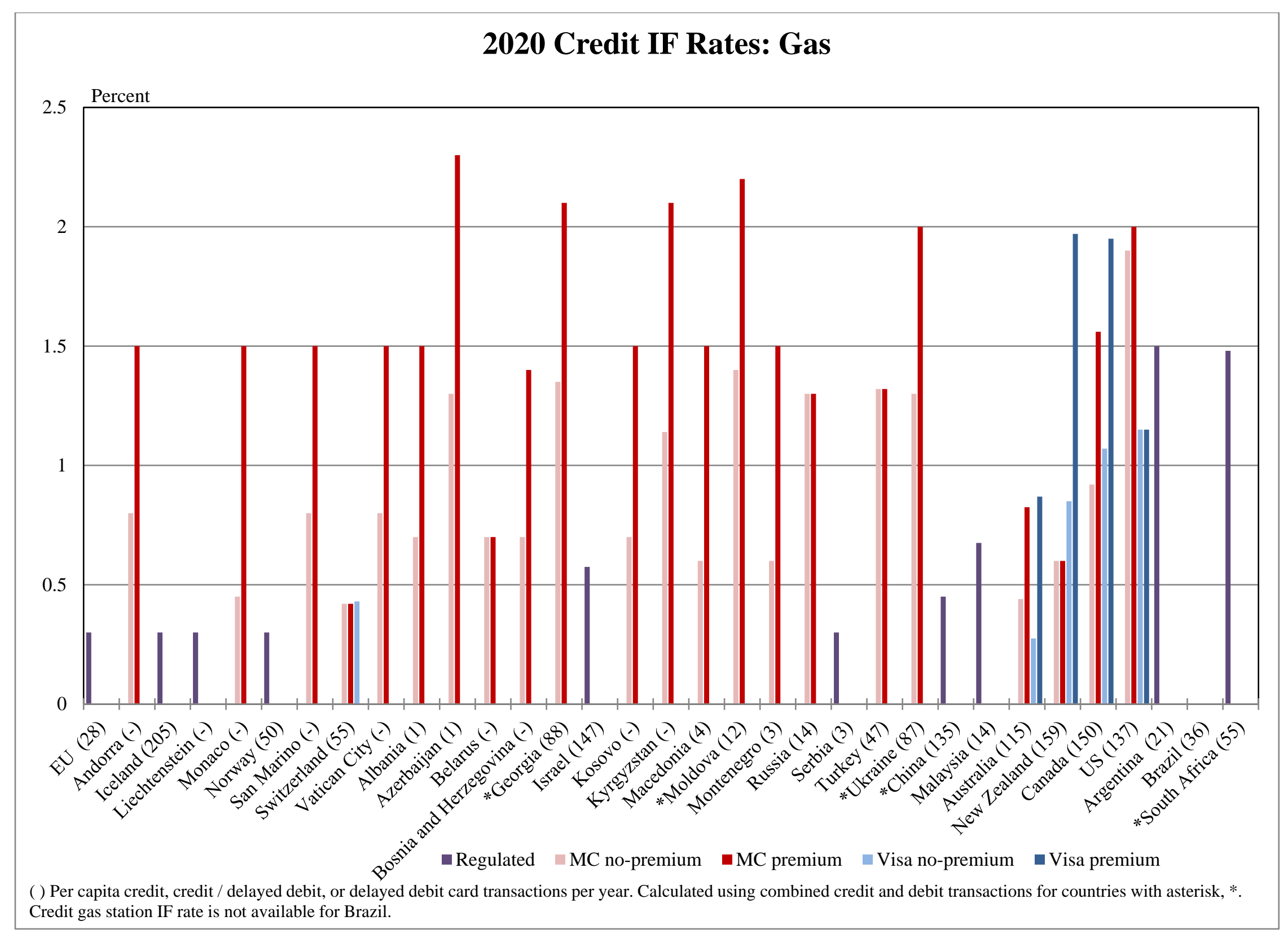




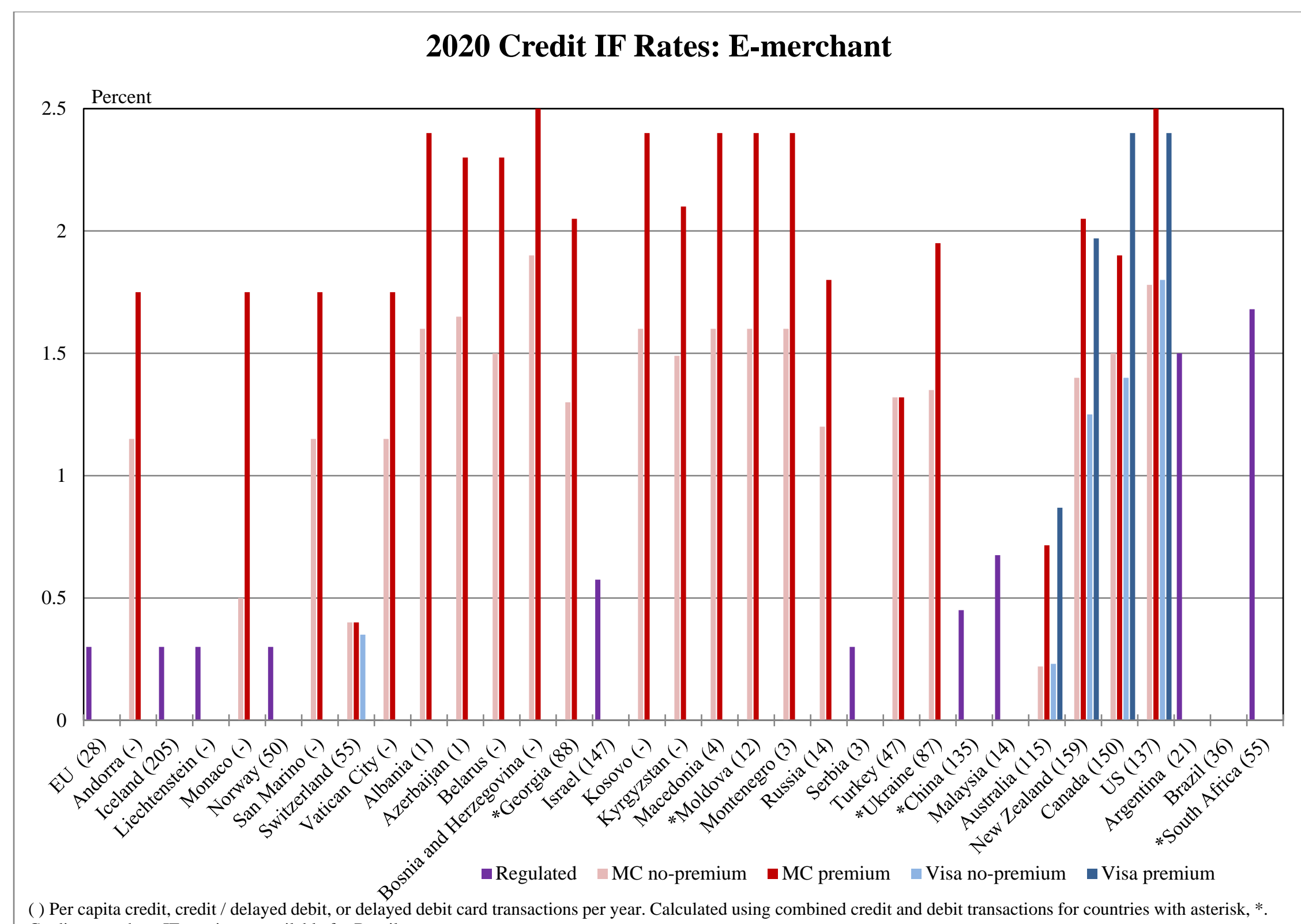

() Per capita credit, credit / delayed debit, or delayed 


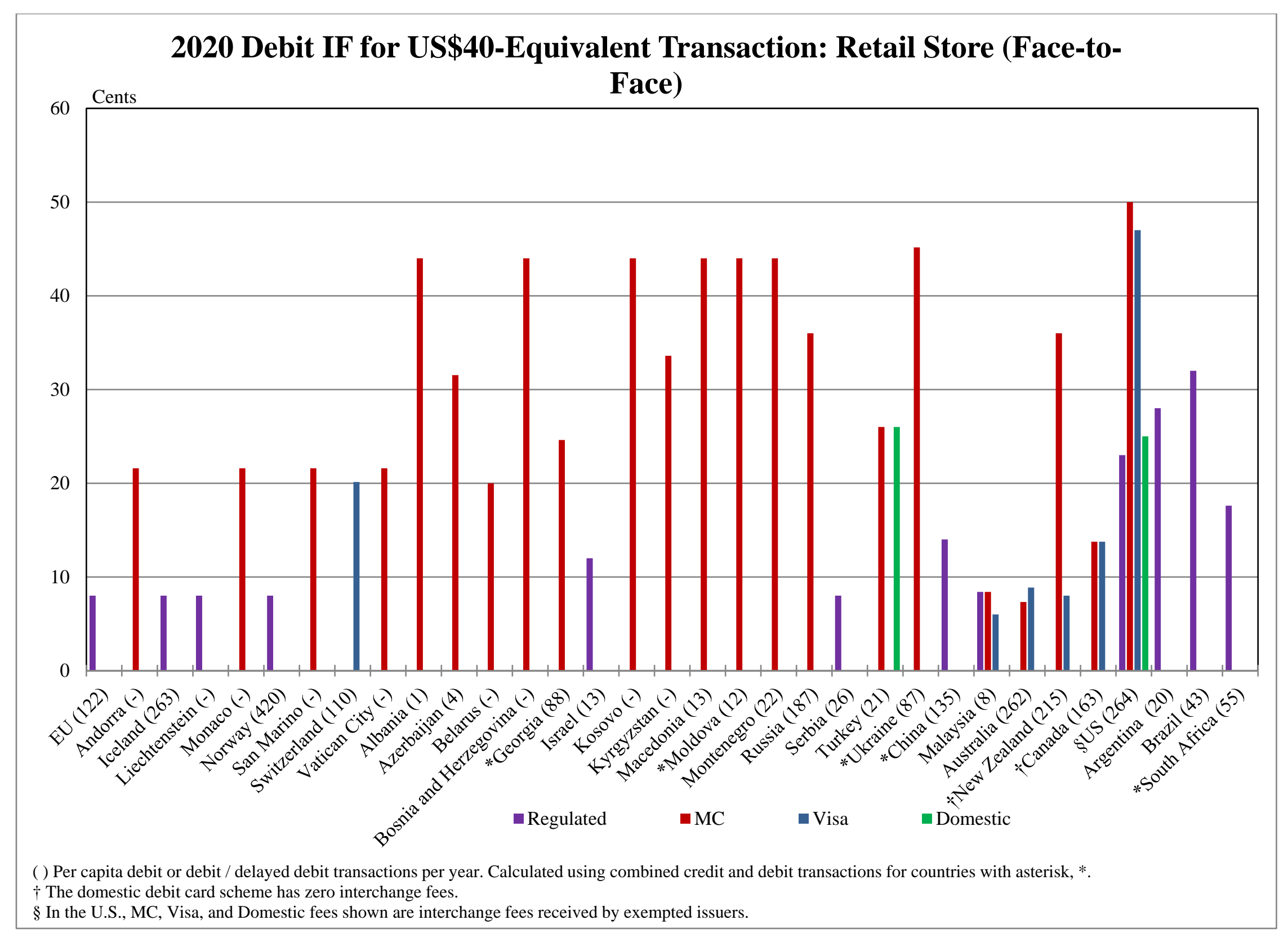




\section{Notes:}

The 2019 average exchange rates are used to convert debit card interchange fees to USD.

As for Mastercard interchange fees, Maestro fees are used for European countries.

As for the U.S. domestic interchange fee, the average PIN debit interchange fee for exempt issuers reported by the Federal Reserve Board is used.

EU countries are: Austria, Belgium, Bulgaria, Croatia, Cyprus, Czech Republic, Denmark, Estonia, Finland, France, Germany, Greece, Hungary, Ireland, Italy, Latvia, Lithuania, Luxembourg, Malta, Netherlands, Poland, Portugal, Romania, Slovakia, Slovenia, Spain, Sweden, and the United Kingdom.

Credit and debit transactions are from 2018 for all countries except Macedonia (2011).

\section{Sources:}

\section{Interchange Fees}

Europe

https://www.visaeurope.com/about-us/interchange-fees/

https://www.mastercard.co.uk/en-gb/about-mastercard/what-we-do/interchange/european-interchange-rates.html

http://www.parlament.gov.rs/49th_Sitting_of_the_Committee_on_Finance,_State_Budget_and_Control_of_Public_Spending.34154.537.html

https://www.boi.org.il/en/NewsAndPublications/PressReleases/Pages/25-2-18.aspx

Asia

https://www.paymentlawadvisor.com/2016/11/28/china-report-uniform-interchange-rates-now-apply-differentiated-interchange-fees-based-on-different-types-ofmerchants-no-longer-exist/

https://www.ocbc.com.my/assets/pdf/Cards/INTERCHANGE_FEE.pdf

\section{Oceania}

https://www.visa.com.au/about-visa/interchange.html

https://www.mastercard.com.au/en-au/about-mastercard/what-we-do/interchange.html

https://www.visa.co.nz/about-visa/interchange.html

https://www.mastercard.co.nz/en-nz/merchants/get-support/merchant-interchange-rates.html

\section{North America}

https://www.mastercard.ca/en-ca/about-mastercard/what-we-do/interchange.html

https://www.visa.ca/dam/VCOM/regional/na/canada/Support/Documents/visa-interchange-rates-april\%202017-published-may-en.pdf

https://www.mastercard.us/en-us/about-mastercard/what-we-do/interchange.html

https://usa.visa.com/dam/VCOM/download/merchants/visa-usa-interchange-reimbursement-fees.pdf

South America

http://www.bcra.gov.ar/mediospago/politica_pagos.asp

https://www.reuters.com/article/us-brazil-cenbank-regulation/brazil-caps-debit-card-fees-may-limit-them-further-idUSKBN1H22XL 
Africa

https://www.resbank.co.za/RegulationAndSupervision/NationalPaymentSystem(NPS)/IDP/Documents/Interchange\%20Project\%20Related\%20Documents/IDP \%20ATM\%20and\%20Card\%20Interchange\%20rates\%20\%20as\%20at\%20March\%202019.pdf

\section{Transactions}

Europe

http://sdw.ecb.europa.eu/browseSelection.do?df=true\&ec=\&dc=\&oc=\&pb=\&rc=\&DATASET=0\&removeItem=\&REF_AREA=105\&node=9689709

https://www.bankofalbania.org/rc/doc/anexx_1_english_in_years_2019_16727.xlsx

https://www.cbar.az/page-45/payment-system-indicators

https://www.cb.is/statistics/statistics/2020/07/14/Payment-intermediation/?stdID=21

https://stats.bis.org/statx/toc/CPMI.html

https://www.norges-bank.no/en/news-events/news-publications/Reports/Norges-Bank-Papers/2019/norges-bank-papers-12019/

https://www.nbs.rs/internet/english/35/statistika/index.html

https://bank.gov.ua/en/news/all/richniy-zvit-natsionalnogo-banku-ukrayini-za-2019-rik

https://www.nbg.gov.ge/uploads/publications/annualreport/2019/annualengfor_web.pdf

http://bnm.md/en/content/annual-report-2018

https://www.cbcg.me/slike_i_fajlovi/eng/fajlovi/fajlovi_publikacije/god_makro_izvjestaj/macroeconomic_report_2018.pdf

https://www.boi.org.il/Lists/BoiChapterTablesFiles/j02b.xls

Asia

https://stats.bis.org/statx/toc/CPMI.html

https://bnm.gov.my/index.php?ch=en_publication\&pg=en_ar\&ac=42\&en

\section{Oceania}

https://stats.bis.org/statx/toc/CPMI.html

http://archive.stats.govt.nz/infoshare/(X(1)S(aekqc2emfnn15m55yjuj21n5))/ViewTable.aspx?pxID=18fbd0b7-9906-42cb-96c7-591b5cd71c14

North America

https://stats.bis.org/statx/toc/CPMI.html

https://www.federalreserve.gov/newsevents/pressreleases/files/2019-payments-study-20191219.pdf

South America

https://stats.bis.org/statx/toc/CPMI.html

Africa

https://stats.bis.org/statx/toc/CPMI.html 
Miscellaneous (population and exchange rate)

http://data.worldbank.org/indicator/SP.POP.TOTL

https://unctadstat.unctad.org/wds/TableViewer/tableView.aspx

https://www.ofx.com/en-us/forex-news/historical-exchange-rates/yearly-average-rates/ 